\title{
COMPETÊNCIAS NO AMBIENTE UNIVERSITÁRIO: A PERCEPÇÃO DOS DISCENTES DA ENGENHARIA DE PRODUÇÃO
}

\author{
Luciana T C de Mello - luciana.mello@ufersa.edu.br \\ Richardson B C Araújo - richardson.bca@outlook.com \\ Luiza L de S Cavalcante - luiza_lorenna@ hotmail.com \\ Lucas A B de Oliveira - lucasambro@ufersa.edu.br \\ Universidade Federal Rural do Semi-Árido, Departamento de Engenharias \\ Rua Gamaliel de Souza, S/N, Alto da Alegria \\ 59515-000 - Angicos - Rio Grande do Norte
}

Resumo: Diante de constantes mudanças no mercado de trabalho, sobretudo na área de engenharia, discute-se muito sobre o desenvolvimento do perfil dos estudantes em formação nos ambientes universitários. As argumentações permeiam as habilidades e competências que precisam ser trabalhadas no decorrer do curso. As habilidades além das técnicas, abrangem aspectos pessoais, como comunicação, inter-relacionamento, liderança de equipes, entre outras. Nesse contexto, esse trabalho possui o objetivo de elencar as competências necessárias ao ambiente universitário na formação do engenheiro de produção, sob perspectiva dos discentes do curso. Para este propósito, a pesquisa possui abordagem quantitativa, método estudo de caso com levantamento do tipo survey. Este, foi aplicado aos discentes do curso de engenharia de produção, com 19 afirmações que exigiam respostas e distribuídas entre 19 afirmações. Como resultado, as competências reconhecidas e confirmadas pelos respondentes foram, Capacidade de Integrar Conteúdos/Práticas, a Capacidade de Persuasão e Argumentação, Capacidade de Receber Opinião, Capacidade de Flexibilização, Capacidade de Disposição para o Novo e Capacidade de Expor a Opinião.

Palavras-chave: Competência. Habilidade. Ambiente universitário. Discente.

\section{INTRODUÇÃO}

A engenharia é uma área profissional criativa estreitamente associada à um perfil de gestão de atividades humanas. Assim engloba de competências científicas, tecnológicas, econômicas a gestão de organizações (SACADURA, 1999). Isto posto, enfatiza-se a necessidade de uma sólida formação técnica pelas Instituições de Ensino Superior (IES) brasileiras, pois foi constatado que engenheiros do país apresentam dificuldades em atitudes práticas como empreendedora, gestão, comunicação, liderança e trabalho em equipes multidisciplinares (MUROFUSHI; BARRETO, 2019) mesmo o setor empresarial compreendendo a área de engenharia com atuação no mercado prático.

Nos ambientes universitários, os educadores frente aos direcionamentos da educação em engenharia passam a exigir e conduzir ações que remodelem as formas de ensino para 
desenvolver as competências exigidas/necessárias ao mercado de trabalho. Neste sentido, desde a década de 90, com a reorganização da economia, em termos monetários, e do estabelecimento da Lei de Diretrizes e Bases da Educação estimulasse o uso de novos experimentos educacionais que conceituados como mais efetivos para auxiliar nas dificuldades (ou falhas, limitações) dos profissionais (BRASIL, 1996).

Nos tempos atuais, é preciso preparar os estudantes para estarem aptos a enfrentar desafios na sua trajetória profissional. Portanto, é imperativo a harmonização de questões técnicas e habilidades práticas materializando os fundamentos técnico-científicos aprendidos, de forma viável e sustentável, conforme preconiza o Capítulo II das Diretrizes Curriculares Nacionais (DCN) do Curso de Graduação em Engenharia. Para isso, passou-se a investigar diferentes abordagens didático-pedagógicas que visassem ao maior aproveitamento do tempo das aulas e melhor rendimento dos discentes - quanto à aprendizagem (ELMOR FILHO et al., 2019).

Porém, para uma preparação satisfatória dos estudantes, inicialmente é necessário discutir o constructo e elencar quais são as habilidades, ou competências, ou ainda as atitudes necessárias repassar aos estudantes de engenharia de hoje - tema de notoriedade atual. Segundo Elmor Filho et al. (2019), autores consideram como habilidades fundamentais a atitude empreendedora, capacidade de gestão e comunicação, instinto de liderança e a possibilidade de trabalhar equipes multidisciplinares, relacionamento interpessoal e atuação sob pressão (GUIMARÃES, BARRETO, 2019) e trabalho em grupo e empatia (CUNHA; BARRETO, 2019). Na literatura, a maioria dos trabalhos que abordam esses fatores, consideram que os próprios estudantes percebem essa necessidade.

Diante do contexto apresentado, este trabalho possui como objetivo elencar as competências necessárias ao ambiente universitário na formação do engenheiro de produção, sob perspectiva dos discentes do curso de uma Instituição de Ensino Superior (IES). Portanto, trata-se de um estudo de caso da engenharia de produção que elenca competências e discute-as sob a ótica de capacidades necessárias ao curso de engenharia de produção de uma universidade pública.

Assim, este artigo é estruturado em quatro seções, além desta de caráter introdutório. A seção dois é uma breve discussão teórica acerca das competências, a seção três é o método de pesquisa e descreve os procedimentos de pesquisa e o instrumento utilizado; a seção quatro discute os resultados e a última seção explicita as conclusões da pesquisa.

\section{AS COMPETÊNCIAS NECESSÁRIAS PARA DISCENTES DO CURSO ENGENHARIA DE PRODUÇÃO}

Conforme as diretrizes dos cursos de graduação em engenharia, espera-se que o engenheiro de produção possua competências e habilidades de integração de fatores de natureza diversificada (tecnológica, humana, empresarial) com vistas ao projeto, implantação, melhoria e manutenção dos sistemas de produção. Entre elas estão a capacidade de trabalhar com equipes multidisciplinares, liderança, planejamento de projetos, flexibilidade, comunicação, criatividade, compreensão e identificação de problemas (econômicos, sociais, administrativos e do meio ambiente), iniciativa empreendedora, disposição de autoaprendizado, compromisso 
e responsabilidade ética profissional, social e ambiental, além de "pensar globalmente, agir localmente" (ABEPRO, 2019).

Para Bazzo e Pereira (2017), além dos conhecimentos específicos devem existir diversos fatores inerentes ao exercício da profissão como aperfeiçoamento contínuo, comunicação, conhecimento objetivo, ética profissional, experimentação, relações humanas e trabalho em equipe. Nessa acepção, as novas Diretrizes Curriculares Nacionais (DCNs) de Engenharia corroboram estes autores discorrendo acerca da necessidade de uma formação generalista que reúna, ao mesmo tempo, as habilidades técnicas com as habilidades "humanas" a fim de acompanhar e as necessidades explícitas e implícitas requisitadas do mercado de trabalho.

Para falar em competências, Teece, Pisano e Shuen (1997), autores mais citados dessa linha, conceituam competências como um conjunto de habilidades que uma organização utiliza para construção e reconfiguração de operações em ambientes de grande dinamicidade. Além desta definição, na visão de Teece, Pisano e Shuen (1997) e Eisenhart e Martin (2000), o vocábulo competências é originário de capacidades, do inglês capability.

Em consonância com Teece, Pisano e Shuen (1997) e Eisenhart e Martin (2000), Winter (2003) discute que o termo capacidade faz referência a um conjunto de habilidades. Assim há uma visão macro que considera dois níveis: nível zero (capacidades operacionais) e nível superior (capacidades dinâmicas) (WINTER, 2003).

As capacidades dinâmicas são as que, estrategicamente, permitem o alcance de um desempenho superior a longo prazo e promovem a reconfiguração das capacidades operacionais para acompanhar as necessidades do mercado (WINTER, 2003). As capacidades operacionais estão relacionadas aos procedimentos de rotina específicos que integram recursos e combinam habilidades e funções dos ativos a nível operacional e que não promovem mudanças (EISENHART; MARTIN, 2000).

$\mathrm{Na}$ linha de capacidades como grandes grupos, algumas são citadas na literatura mais comumente, como capacidade de aprendizagem (KANNINEN et al., 2017), capacidade de adaptação (SPRING; ARAUJO, 2009), capacidade de inovação (LIN et al., 2011) e a capacidade de integração (DATTA; ROY, 2011).

A capacidade de aprendizagem (KANNINEN et al., 2017), pode ser vista como a capacidade de aprender novas funções. A capacidade de adaptação (SPRING; ARAÚJO, 2009) acompanha as condições do mercado em cada contexto específico e promove alterações internas. A capacidade de inovação (LIN et al., 2011) significa melhorar a experiência do cliente com novas variedades de soluções. A capacidade de integração (DATTA; ROY, 2011) busca o desenvolvimento de um relacionamento entre os envolvidos nas operações, criando uma linguagem comum para facilitar os serviços e contribuir para a operação macro.

Quanto à intenção de relacionar as competências utilizadas na literatura para organizações do mercado e as competências buscadas pelo mercado empresarial nos engenheiros, o Quadro 1 sugere um possível alinhamento.

Quadro 1- Capacidade para o engenheiro

\begin{tabular}{|c|c|c|}
\hline $\begin{array}{c}\text { Capacidades } \\
\text { Dinâmicas }\end{array}$ & Capacidades Operacionais & Competências para o Engenheiro de Produção \\
\hline $\begin{array}{c}\text { Capacidade de } \\
\text { Aprendizagem }\end{array}$ & Capacidade de aprender novas funções & $\begin{array}{c}\text { Capacidade de aprender conhecimentos (técnicos } \\
\text { e humanos) }\end{array}$ \\
\hline
\end{tabular}




\section{Evento On-line}

\begin{tabular}{|c|c|c|}
\hline $\begin{array}{l}\text { Capacidades } \\
\text { Dinâmicas }\end{array}$ & Capacidades Operacionais & Competências para o Engenheiro de Produção \\
\hline & Capacidade de estabelecer parcerias & Capacidade de trabalhar em equipe \\
\hline & $\begin{array}{l}\text { Capacidade de compartilhar } \\
\text { informações e conhecimento }\end{array}$ & Capacidade de aprimoramento contínuo \\
\hline \multirow{4}{*}{$\begin{array}{l}\text { Capacidade de } \\
\text { Adaptação }\end{array}$} & $\begin{array}{l}\text { Capacidade de desenvolver nova } \\
\text { mentalidade }\end{array}$ & Capacidade de disposição para o novo \\
\hline & Capacidade de flexibilizar & $\begin{array}{c}\text { Capacidade de redistribuição e delegação de } \\
\text { tarefas }\end{array}$ \\
\hline & $\begin{array}{c}\text { Capacidade de avaliar ambientes e } \\
\text { riscos }\end{array}$ & $\begin{array}{l}\text { Capacidade de atuação em situações e contextos } \\
\text { diversos }\end{array}$ \\
\hline & Capacidade de mudanças & Capacidade de se renovar \\
\hline \multirow{2}{*}{$\begin{array}{l}\text { Capacidade de } \\
\text { Inovação }\end{array}$} & $\begin{array}{l}\text { Capacidade de desenvolver novos } \\
\text { produtos e serviços }\end{array}$ & $\begin{array}{l}\text { Capacidade de identificar oportunidades de } \\
\text { melhorias (produtos, processos e serviços) }\end{array}$ \\
\hline & Capacidade de personalização & $\begin{array}{l}\text { Capacidade de desenvolver e implementar } \\
\text { estratégias de solução de problemas }\end{array}$ \\
\hline \multirow{2}{*}{$\begin{array}{l}\text { Capacidade de } \\
\text { Integração }\end{array}$} & $\begin{array}{c}\text { Capacidade de identificação das } \\
\text { necessidades dos clientes }\end{array}$ & Capacidade de identificar novas oportunidades \\
\hline & $\begin{array}{l}\text { Capacidade de ouvir a opinião dos } \\
\text { clientes para melhoria interna }\end{array}$ & $\begin{array}{c}\text { Capacidade de motivação, liderança e gestão de } \\
\text { pessoas }\end{array}$ \\
\hline
\end{tabular}

Fonte: Adaptado de Mello e Araújo (2019)

\section{MÉTODO DE PESQUISA}

No contexto deste trabalho, foram realizados dois procedimentos de pesquisa. O primeiro, uma revisão de literatura acerca dos temas: capacidades dinâmicas e operacionais, novas DCNs de Engenharia e as competências do engenheiro de produção consoante a Associação Brasileira de Engenharia de Produção (ABEPRO) a fim de correlacioná-los, conforme explicitado no Quadro 1. O segundo procedimento compreendeu a realização de uma survey junto ao corpo discente do curso de graduação em engenharia de produção, apresentado na seção 3.1.

Assim, quanto ao método de pesquisa, o presente estudo classifica-se com abordagem quantitativa, descritiva. O método de pesquisa adotado é o estudo de caso com levantamento tipo survey, visto aos objetivos supracitados de avaliação de acontecimentos com variáveis já definidas (MIGUEL; HO, 2012). Além de investigar relações de causalidade das incógnitas acometendo em condições para replicação e generalização do estudo (MARTINS, 2012).

\subsection{Elaboração e aplicação do instrumento de pesquisa}

A elaboração do questionário seguiu as variáveis da literatura discutidas na seção 2, e elencadas no Quadro 1. Vale salientar que, na utilização do questionário respondido por discentes do curso as capacidades operacionais foram adaptadas para que ficassem mais coerentes com o contexto universitário. A Figura 1 retoma as variáveis e seus respectivos códigos para facilitar as análises. Tem-se cinco grandes grupos de capacidades: A - Capacidade de Aprendizagem, B - Capacidade de Adaptação, C - Capacidade de Inovação, D - Capacidade de Integração e E - Capacidade de Comunicação. 


\section{Evento On-line}

Figura 1 - Questionário aplicado

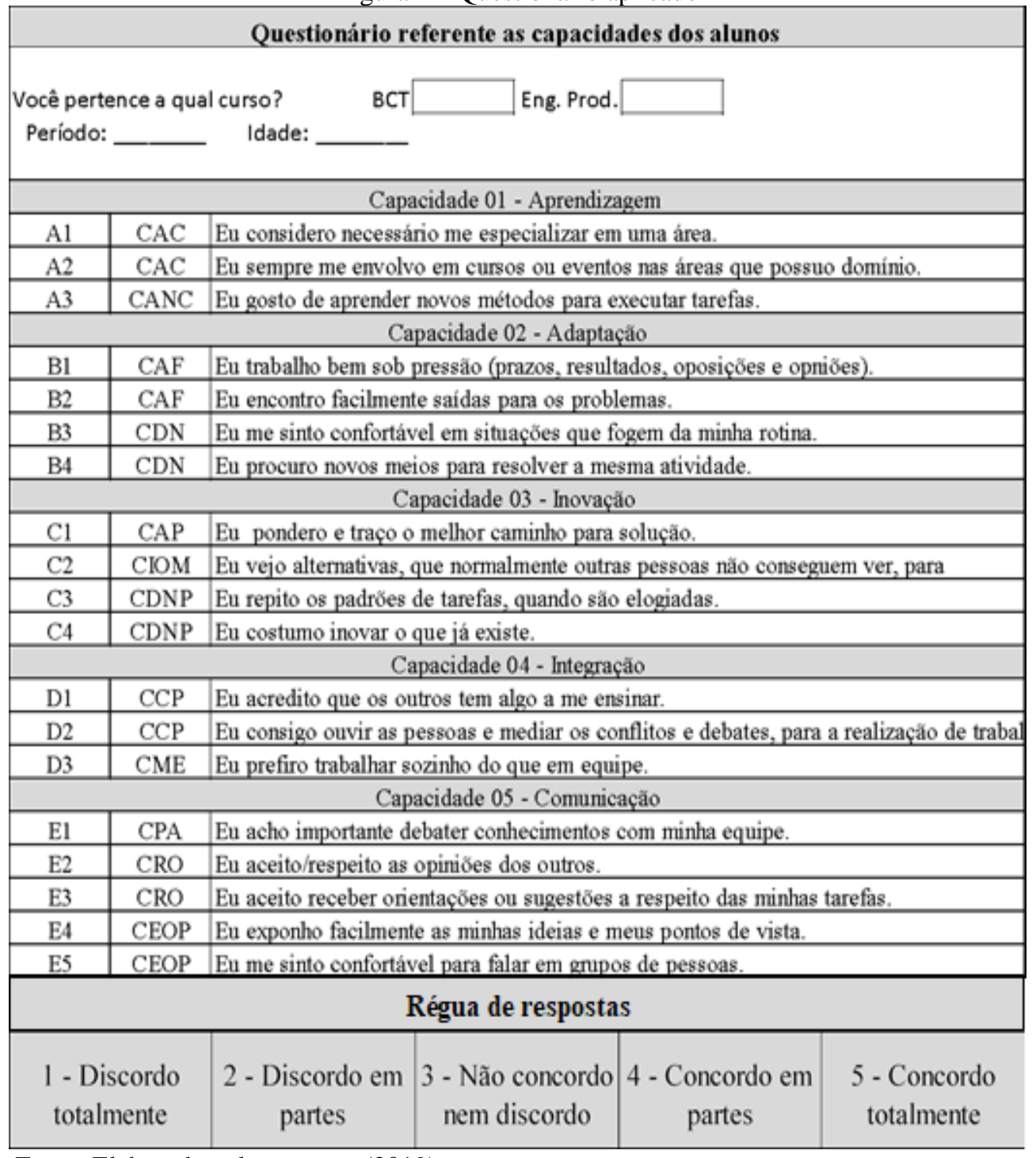

Fonte: Elaborado pelos autores (2019)

Legenda: CAC - Capacidade de Aprimoramento Contínuo; CANC - Capacidade de Aprender Novos Conhecimentos; CAF Capacidade de Flexibilização; CDN - Capacidade de Disposição para o Novo; CAP - Capacidade de Personalizar; CIOM Capacidade de Identificar Oportunidades de Melhorias; CDNP - Capacidade de Desenvolver e Implementar Novas Práticas; $C C P$ - Capacidade de Integrar Conteúdos/Práticas; CME - Capacidade de Integrar Membros da Equipe; CPA - Capacidade de Persuasão e Argumentação; CRO - Capacidade de Receber Opinião; CEOP - Capacidade de Expor a Opinião.

O instrumento de pesquisa, pode ser dividido em duas etapas: a primeira traça o perfil dos respondentes e a segunda o levantamento das capacidades, entendidas neste estudo como competências, do tipo survey.

O delineamento do perfil dos estudantes é de caráter básico com informações do período do curso e a idade. Já o levantamento, em estilo survey, possui afirmações e uma régua de respostas com intervalo de 1 a 5, conforme modelo ilustrado na Figura 1. As afirmações foram desenvolvidas com base nas variáveis de Capacidades Operacionais. Para cada variável foi 


\section{OCOBENGE C. COBENCE $2020 \quad \begin{aligned} & \text { de Educaçäo em Engenharia } \\ & \text { da ABENGE }\end{aligned}$}

\section{Evento On-line}

necessária, mas não restrito, pelo menos uma afirmação. Por isto, o questionário apresentado é formado por 19 afirmações que representam 12 Capacidades Operacionais.

\section{DISCUSSÃO DOS RESULTADOS}

$\mathrm{Na}$ caracterização do perfil dos respondentes (período do curso e idade), a maioria dos respondentes $(80 \%)$ estavam no último ano do curso $\left(9^{\circ}\right.$ e $10^{\circ}$ períodos $)$, na faixa etária entre 22 e 25 anos de idades.

Em relação às informações do curso, apesar de a pesquisa abranger apenas o curso de Engenharia de Produção é importante destacar que o curso é de segundo ciclo, no qual os alunos só fazem a entrada oficial depois de terem feito o curso de Bacharel em Ciência e Tecnologia (BCT). Porém, na transição de BCT para Engenharia de Produção é possível que os estudantes ainda se encontrem oficialmente matriculados no curso de BCT, mesmo que tenham optado pelo curso de Engenharia de Produção. Assim, eles podem estar matriculados em disciplinas do curso de Engenharia de Produção, cursando o último ano de BCT, e estes também foram considerados respondentes. Por este motivo, foi necessário compreender a qual curso faziam parte oficialmente e em qual período estavam.

Ainda, em análise ao perfil discente, para este trabalho é relevante para a pesquisa os respondentes de final do curso porque pressupõem-se que eles demonstrem maior maturidade e entendimento sobre os aspectos respondidos - já que estão mais próximos de ingressar no mercado de trabalho. Nessa lógica, as Figuras 2 e 3 mostram as respostas sobre o período do curso e idade.

Figura 2 - Período do curso dos respondentes

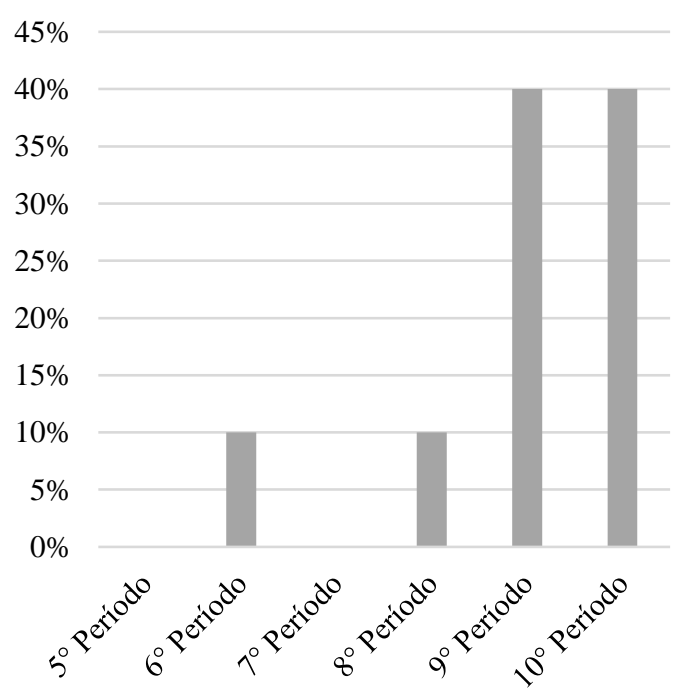

Fonte: Dados da pesquisa (2020)
Figura 3 - Faixas etárias dos respondentes

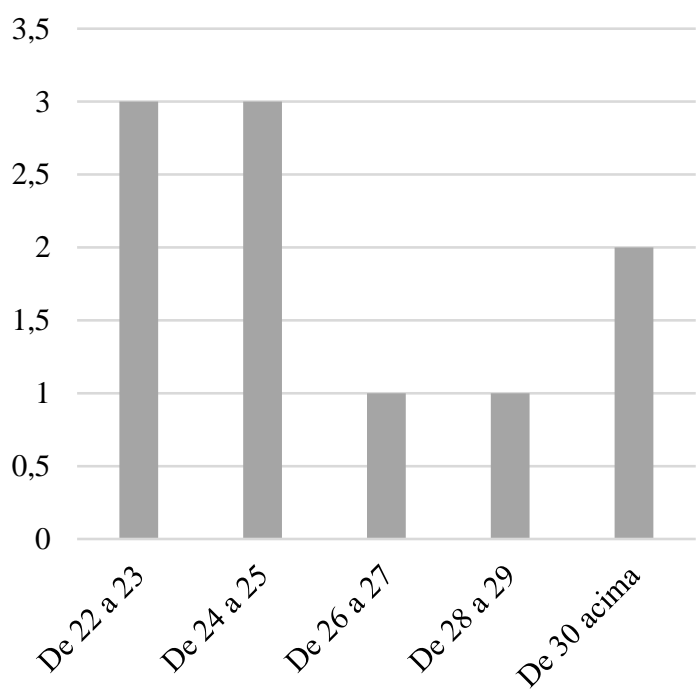

Fonte: Dados da pesquisa (2020) 


\section{Evento On-line}

Sobre as Capacidades, no qual os respondentes avaliaram afirmações de acordo com uma numeração "em régua" com intervalo de 1 a 5 , as respostas estão em formato de gráfico na Figura 4. Vale salientar que as 19 afirmações estão representadas por códigos no quadro, mas foram descritas na seção de Método de Pesquisa.

Figura 4 - Respostas dos discentes

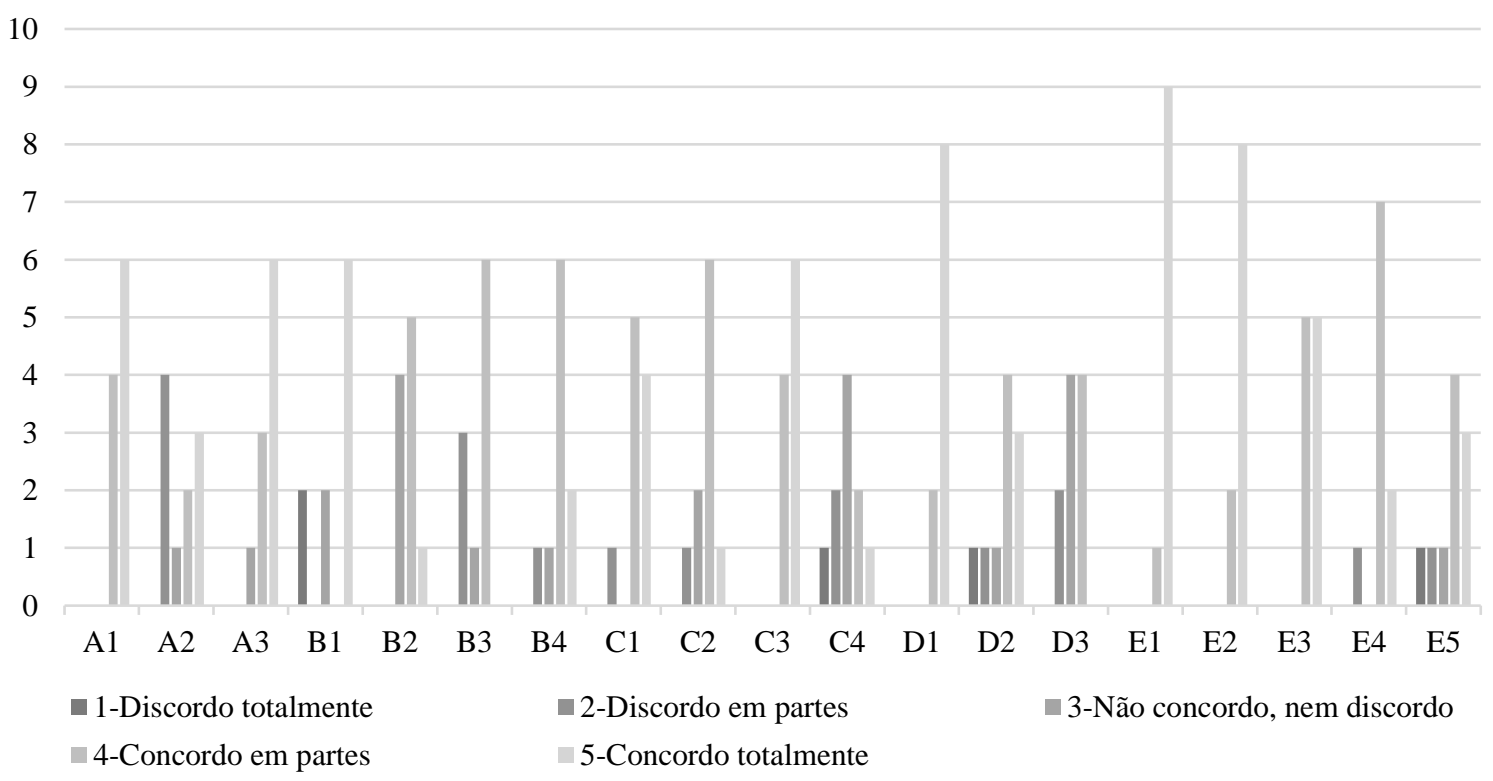

Fonte: Dados da Pesquisa (2020)

De acordo com a Figura 4, as afirmações que obtiveram a maioria das respostas como o número "5 - Concordo totalmente" foram as A1, A3, B1, C3, D1, E1 e E2. Em algumas afirmações, a principal resposta foi "4-Concordo em partes", as capacidades B2, B3, B4, C1, C2, D2, E4 e E5. Já para as respostas "1-Discordo totalmente" e "2-Discordo em partes", a capacidade A2 teve a maioria de respondentes, mas a capacidade B3 também teve um número considerável de respondentes, mesmo que a maioria tenha "concordado em partes".

Com essa análise, presume-se que a Capacidade de Aprimoramento Contínuo (CAC) que considera cursos e eventos para aprender não é vista como tão importante para os estudantes de engenharia. Por outro lado, a Capacidade de Integrar Conteúdos/Práticas (CCP), a Capacidade de Persuasão e Argumentação (CPA) e a Capacidade de Receber Opinião (CRO) são reconhecidas pelos discentes respondentes como de grande importância.

\section{CONCLUSÕES}

A retomada do objetivo deste trabalho de elencar as competências necessárias ao ambiente universitário na formação do engenheiro de produção, em uma Instituição de Ensino Superior, sob perspectiva dos discentes do curso, permite afirmar seu alcance com a aplicação da survey. As competências foram elencadas a partir da literatura, adaptadas para a Survey e confirmadas

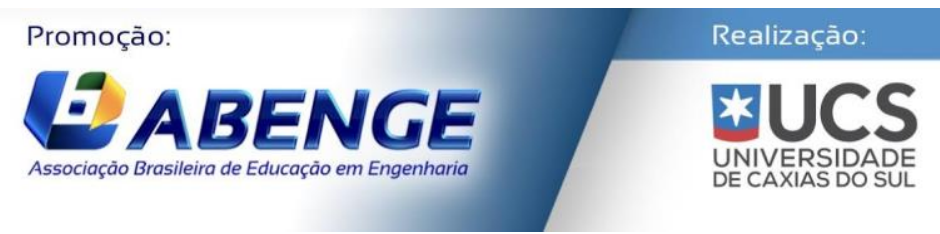


pelos respondentes. São elas: a Capacidade de Integrar Conteúdos/Práticas (CCP), a Capacidade de Persuasão e Argumentação (CPA), a Capacidade de Receber Opinião (CRO), a Capacidade de Flexibilização (CCP), a Capacidade de Disposição para o Novo (CDN) e a Capacidade de Expor a Opinião (CEOP).

Dessarte, é enfatiza-se a relevância de estudos como estes que buscam compreender as diversas e mais pertinentes competências e habilidades necessárias a formação do futuro engenheiro, em razão de sua atuação em um mercado cada vez mais dinâmico. Como limitação desta pesquisa, ainda que em consonância com o objetivo delineado, cita-se aplicação da survey com discentes de apenas um curso. Fato, que converge para as sugestões de estudos posteriores, então sugere-se a ampliação e replicação deste para outros cursos e contextos.

Além da sugestão de ampliação e replicação da pesquisa, também se explicita a necessidade de estudos que abordem a percepção de docentes e gestores da engenharia de produção e de outras áreas com possibilidade de interdisciplinaridade.

\section{REFERÊNCIAS}

ABEPRO. Associação Brasileira de Engenharia de Produção. Um panorama da Engenharia de Produção. Disponível em: <http://www.abepro.org.br/interna.asp?ss=1\&c=924> Acesso em: 24 abr 2019.

BAZZO, Walter Antonio; PEREIRA, Luiz Teixeira do Vale. Introdução à Engenharia: Conceitos, ferramentas e comportamentos, $4^{\mathrm{a}}$ edição, Florianópolis: Editora UFSC, 2017.

BRASIL. Conselho Ministério da Educação e dos Desportos. Lei de Diretrizes e Bases da Educação Nacional. Lei n. 9. 394 de 20 de dezembro de 1996.

CUNHA, Maria Angélica Silva; BARRETO, Maria Auxiliadora Motta. Avaliação de competências transversais em disciplina integradora empresa-universidade. In: Congresso Brasileiro de Educação em Engenharia, Fortaleza CE, 2019.

DATTA, Partha Priya; ROY, Rajkumar. Operations strategy for the effective delivery of integrated industrial product-service offerings: two exploratory defence industry case studies. International Journal of Operations \& Production Management, v. 31, n. 5, p. 579-603, 2011.

EISENHARDT, Kathleen. M.; MARTIN, Jeffrey A. Dynamic capabilities: what are they? Strategic Management Journal, v. 21, p. 1105-1121, 2000.

ELMOR FILHO, Gabriel et al. Uma nova sala de aula é possível: aprendizagem ativa na educação em engenharia. 1. ed. Rio de Janeiro: LTC, 2019. 


\section{COBENCE $2020 \begin{aligned} & \text { de Educaçäo em Engenharia } \\ & \text { da ABENGE }\end{aligned}$}

"Os desafios para formar hoje o engenheiro do amanhã"

GUIMARÃES Melina Murgel; BARRETO Maria Auxiliadora Motta. Desenvolvimento de competências transversais em cursos de engenharia na percepção de estudantes brasileiros e dinamarqueses. In: Congresso Brasileiro de Educação em Engenharia, Fortaleza CE, 2019.

KANNINEN, Tiina et al. Exploring the dynamic capabilities required for servitization: the case process industry. Business Process Management Journal, v. 23, n. 2, p. 226-247, 2017.

LIN, Yong et al. Servitization strategy: strategic priority, capacity requirement, and organizational feature. In: INTERNATIONAL CONFERENCE IN SERVICE OPERATIONS, LOGISTICS, AND INFORMATICS (SOLI), 2011. Anais... 2011.

MELLO, Luciana Torres Correia de, ARAÚJO, Richardson Bruno Carlos. As capacidades dinâmicas e operacionais no ambiente universitário In: Congresso Brasileiro de Educação em Engenharia, 47, Anais eletrônicos..., Fortaleza, 2019.

MARTINS, Roberto Antonio. Abordagens quantitativa e qualitativa. In: MARTINS, Roberto. Antonio (Org.). Metodologia de pesquisa em engenharia de produção e gestão de operações. 2. ed. Rio de Janeiro: p. 47-56, 2012.

MIGUEL, Paulo Augusto Cauchik.; HOO, Linda Lee Levantamento tipo survey. In: MARTINS, Roberto Antonio (Org.). Metodologia de pesquisa em engenharia de produção e gestão de operações. [s.l: s.n.]. p. 93, 2012.

MUROFUSHI, Juliana. BARRETO, Maria A. M. Educação 4.0 na engenharia: percepção dos docentes de 3 universidades brasileiras. In: Congresso Brasileiro de Educação em Engenharia, 47, Anais eletrônicos... Fortaleza, 2019.

SACADURA, Jean-François. A formação do engenheiro no limiar do terceiro milênio. In: LINSENGEN, Irlan Von et al. (Org.). Formação do engenheiro: desafios da atuação docente, tendências curriculares e questões da educação tecnológica. Florianópolis, UFSC.

SPRING, Martin; ARAUJO, Luis. Service, services and products: rethinking operations strategy. International Journal of Operations \& Production Management, v. 29, n. 5, p. 444-467, 2009.

TEECE, David J.; PISANO, Gary, SHUEN, Aamy. Dynamic capabilities and strategic management. Strategic Management Journal, v. 18, n. 7, p. 509-533. 1997.

WINTER, Sidney G. Understanding dynamic capabilities. Strategic Management Journal. v. 24, p. 991-995, 2003. 


\title{
Evento On-line
}

\section{COMPETENCES IN THE UNIVERSITY ENVIRONMENT: THE PERCEPTION OF DISCENTS OF PRODUCTION ENGINEERING}

\begin{abstract}
In the face of constant changes in the job market, especially in the area of engineering, much has been discussed about the development of the profile of students who are being trained in university environments. Discussions permeate the skills and competences that must be worked on during the course. The capabilities go beyond techniques, covering personal aspects, such as communication, inter-relationship, team leadership, among many others. In this context, this work aims to list the capabilities necessary for the university environment in the training of the industrial engineer, from the perspective of the students of the course. To achieve the objective, the research, with the quantitative approach and survey method, was carried out by applying a questionnaire to the students of the industrial engineering course, with statements that required responses on an ordinal scale from 1 to 5 , in which 12 capabilities were taken into account, distributed among 19 statements. As a final result, the capabilities recognized and confirmed by the respondents were Capability to Integrate Content/Practices, Capability of Persuasion and Argumentation, Capability to Receive Opinion, Capability of the Flexibility, Capability of Willingness to the New, and Capability of to Expose the opinion.
\end{abstract}

Keywords: competence, ability, capability, student, university. 